\title{
Experimental investigation of stationary concepts in cyclic duopoly games
}

\author{
Sebastian J. Goerg • Reinhard Selten
}

Received: 10 July 2007 / Accepted: 11 June 2009 / Published online: 24 June 2009

(C) The Author(s) 2009. This article is published with open access at Springerlink.com

\begin{abstract}
We experimentally test the predictive success of three stationary concepts in two cyclic duopoly games. The concepts are Nash equilibrium, impulse-balance equilibrium and payoff-sampling equilibrium. In the experiment 11 independent subject groups, consisting out of six participants interacting over 200 rounds, were gathered for each game. The comparison of the three concepts with mixed strategies shows that the order of performance from best to worst is as follows: payoff-sampling equilibrium, impulse-balance equilibrium, and Nash equilibrium. In addition the data exhibit a weak but significant tendency over time in the direction of coordination at a pure strategy equilibrium.
\end{abstract}

Keywords Cyclic game duopoly experiment · Impulse-balance equilibrium · Payoff-sampling equilibrium

JEL Classification C73 $\cdot$ D43 · C90

\section{Introduction}

Several studies have demonstrated that the concept of mixed Nash equilibrium fails to explain observed behavior in experiments (e.g., Brown and Rosenthal 1990; Erev and

Electronic supplementary material The online version of this article (http://dx.doi.org/10.1007/s10683-009-9218-8) contains supplementary material, which is available to authorized users.

S.J. Goerg $(\bowtie)$

Max Planck Institute for Research on Collective Goods, Kurt-Schumacher-Str. 10, 53113 Bonn,

Germany

e-mail: goerg@coll.mpg.de

S.J. Goerg · R. Selten

BonnEconLab, Laboratory for Experimental Economics, Department of Economics,

University of Bonn, Adenaueralle 24-42, 53113 Bonn, Germany 
Roth 1998, and Avrahami et al. 2005). In a recent paper by Selten and Chmura (2008) on completely mixed $2 \times 2$ games played by small populations with random matching over 200 rounds, several behavioral stationary concepts beat the Nash equilibrium in predicting subjects' behavior. In that study the two behavioral concepts of impulsebalance equilibrium and payoff-sampling equilibrium proved to be successful but hard to differentiate.

The main goal of this paper is to examine the results from Selten and Chmura in a different environment. We therefore compare the predictive success of Nash equilibrium, impulse-balance equilibrium and payoff-sampling equilibrium in two cyclic games.

The notion of a cyclic game has been introduced by Selten and Wooders (2001). Cyclic games can be applied to simplified recurring situations with intertemporal competition. Similar situations are investigated by overlapping generations models (starting with Diamond 1965), but on the basis of market theory rather than game theory. In our study we applied the concept of a cyclic game to the market entry situation in a duopoly.

In our duopoly games exactly one potential entrant in each period can decide whether he wants to enter the market or not. If he enters he stays in the market for exactly two periods and then exits. Imagine that this market is very narrow. A firm makes a negative profit if it has to share the market with another firm in both periods. A positive profit can be obtained if the firm is the only supplier in at least one of the two periods.

In these games it is always profitable to enter an empty market. However, a potential entrant who faces an occupied market in period $t$ has to think about the consequences of entering the market. If he enters, the following potential entrant in period $t+1$ will also face an occupied market and will find himself in the same decision problem. As we shall see this leads, for each of the three stationary concepts, to a different symmetric mixed equilibrium, in which every player facing an occupied market enters with the same probability.

We will investigate two different cyclic games, which share two features with the $2 \times 2$ games investigated by Selten and Chmura (2008): First, two types of players compete against each other and, second, both player types have two possible actions to choose from. However, the investigated cyclic games differ by three properties from the $2 \times 2$ games from Selten and Chmura (2008): First, in our cyclic games the payoffs of both players are symmetric, while in Selten and Chmura (2008) they were not. Second, players in cyclic games have perfect information in the sense that each information set consists of only one node. In cyclic games the players act sequentially in contrast to the $2 \times 2$ games with simultaneous decisions. Third, while in the mentioned $2 \times 2$ games only mixed equilibria existed there are two pure strategy equilibria in the cyclic duopoly games.

Because of the similarities and the differences we think that the cyclic game is a good starting point to observe the concepts investigated by Selten and Chmura in a different environment and a good way to identify potential reasons for alternative results.

Our results confirm the superior predictive power of impulse-balance equilibrium and payoff-sampling equilibrium in comparison to Nash equilibrium. Overall, both 
behavioral concepts perform significantly better than Nash equilibrium does. Our results differ from Selten and Chmura (2008) regarding the equality of impulse-balance equilibrium and payoff-sampling equilibrium: In our study payoff-sampling equilibrium performs significantly better than impulse-balance equilibrium does. In our opinion this disadvantage of impulse-balance equilibrium in comparison to the mentioned $2 \times 2$ games is caused by the sequential move structure of the cyclic game.

As mentioned before, our experimental setup also permits asymmetric equilibria in which one of the two players, say player one, always enters and the other player never enters. In fact the data reveal a significant tendency towards convergence to pure strategies over time. This finding sheds some doubt on the comparison of the three stationary concepts in the long run.

The rest of the paper is organized as follows: in Sect. 2 we will describe and explain the cyclic oligopoly game in more detail. Afterwards the three stationary concepts are introduced, applied to the games and their predictions are derived. In Sect. 4 the experimental procedure is described and subsequently, Sect. 5 gives our results. Finally, Sect. 6 summarizes and concludes this paper.

\section{The cyclic game}

A cyclic game may be looked upon as a condensed description of an infinite game. In the following we shall describe the infinite game structure underlying our experiment. The game runs over periods $t=1,2, \ldots$ In each period $t$ a potential entrant $t$ has to decide whether he enters the market or not in period $t$. If he enters he stays in the market for periods $t$ and $t+1$ and exits then. A potential entrant $t$ may face an empty market, which is always true for $t=1$. For $t=2,3, \ldots$ the market is empty at period $t$ if the potential entrant $t-1$ did not enter the market. The market is occupied for $t$ with $t=2,3, \ldots$ if the potential entrant $t-1$ did enter the market.

The concept of a cyclic game permits a condensed description of game situations like the one investigated in our experiment. In our case, the cyclic game has only two players. These two players are roles of the potential entrants. The odd-numbered members of an independent subject group are in the role of player 1 and the evennumbered are in the role of player 2.

Figure 1 illustrates the structure of the cyclic duopoly game as introduced by Selten and Wooders (2001). The figure has the structure of a directed graph with 3 types of nodes and additional information regarding player and payoff. Each point describes a situation of either player 1 or player 2 . At a decision point a player has to decide between two alternatives in our case IN or OUT. Here IN means entering and OUT means not entering. At a payoff point or an exit and payoff point, the payoff of the concerning player is shown in rectangular brackets above this point. The arrows show the direction in which the game moves from one situation to the next.

At the upper right corner player 1 can decide between IN and OUT in the situation of an empty market. In the case OUT he receives a payoff of $W$ at a payoff and exit point. If he chooses IN he receives a payoff of $U$ in this period and the game moves to a decision point of player 2. If player 2 then chooses OUT first, a payoff and exit point is reached, at which player 2 receives $W$ and then a payoff and exit point of 


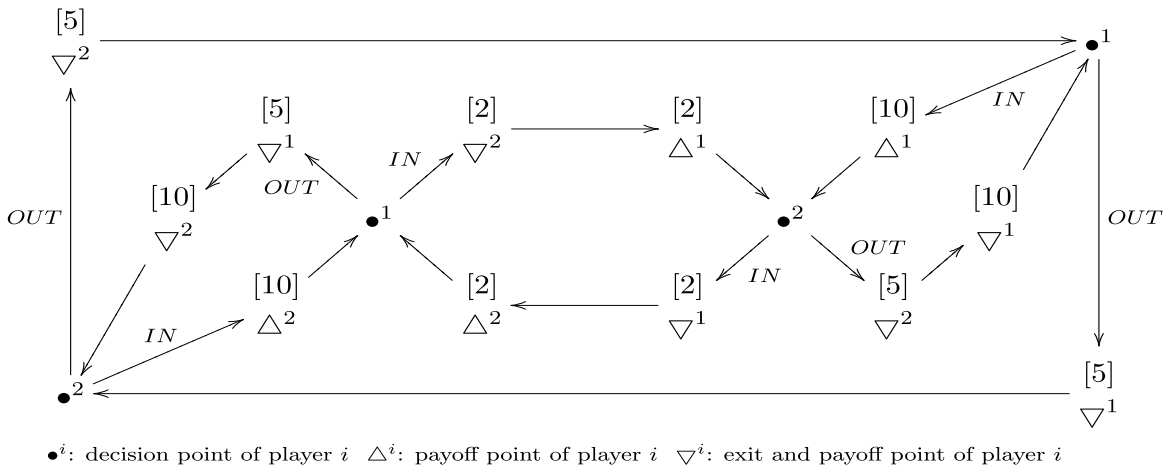

Fig. 1 Structure of the cyclic duopoly game

Table 1 Parameter for $U, V$ and $W$ in Game A and Game B

\begin{tabular}{llll}
\hline & $U$ & $V$ & $W$ \\
\hline Game A & 10 & 2 & 5 \\
Game B & 10 & 3 & 9 \\
\hline
\end{tabular}

player 1, where he receives $U$. From there the game moves back to the upper right corner. The other parts of the figure are to be understood in the same way.

In our cyclic oligopoly game $U$ is the payoff for a period in which the entrant is alone in the market, $V$ is the payoff for a period in which the entrant is not alone in the market, and $W$ is the payoff for two periods if the market is not entered. The parameters $U, V$ and $W$ were chosen in such a way that the condition $U+V>W>$ $2 V$ is satisfied and thus implies $U>V$. Therefore entering an empty market always leads to a higher payoff than not entering an empty market. In our experiments we applied two different sets of the parameters $U, V$ and $W$, which are given in Table 1 .

These different parameter sets lead to different equilibria and provide different attractiveness for not entering an occupied market. ${ }^{1}$ In Game B the payoff for not entering the market is higher than in Game A; therefore it should lead to a smaller entry rate in Game B compared to the one in Game A.

\section{Three stationary concepts for the cyclic game}

We shall look at three stationary concepts: Nash equilibrium, impulse-balance equilibrium and payoff-sampling equilibrium. First, we will derive the Nash equilibria for the two games. Then we will explain the basic ideas behind the two behavioral concepts, impulse-balance equilibrium and payoff-sampling equilibrium.

As has been shown in Selten and Wooders (2001) the cyclic game has three Nash equilibria. A symmetric mixed-strategy equilibrium and two pure-strategy equilibria.

\footnotetext{
${ }^{1}$ In addition we chose the parameters to ensure that the distances between the resulting equilibria are big enough to determine the concept with the best fit to the data.
} 
In the symmetric mixed equilibrium the probability $\alpha$ for entering if the market is occupied is as follows:

$$
\alpha=\frac{U+V-W}{U-V}
$$

In Game A this leads to the probability of entering the occupied market of $\alpha=0.875$ and in Game B to the probability of $\alpha=0.571$. If the market is empty the probability of entering is always one. This is also true for the two pure equilibria. However, there the probabilities $\alpha_{1}$ and $\alpha_{2}$ are

$$
\alpha_{1}=1 \quad \text { and } \quad \alpha_{2}=0
$$

in the first pure-strategy equilibrium and

$$
\alpha_{1}=0 \quad \text { and } \quad \alpha_{2}=1
$$

in the second pure-strategy equilibrium.

We now turn our attention to the impulse-balance equilibrium. Impulse-balance equilibrium is based on the idea of learning direction theory (Selten and Buchta 1999), which looks at probabilities of decisions as behavioral tendencies. Selten and Buchta explain the concept by the example of a marksman aiming at a trunk: "If he misses the trunk to the right, he will shift the position of the bow to the left and if he misses the trunk to the left he will shift the position of the bow to the right. The marksman looks at his experience from the last trial and adjusts his behavior [...]." (p. 86, Selten and Buchta 1999)

The concept of impulse-balance equilibrium (Selten et al. 2005, and Selten and Chmura 2008) models these adjustments with impulses received after the realization of payoffs. Suppose that the first of two strategies has been chosen in a period and this strategy was not the best reply to the strategy played by the other player. Then the player receives an impulse towards the second strategy. This impulse is the difference between the payoff the player could have received for his best reply minus the payoff actually received given the strategy used by the other player in this period. The player does not receive an impulse if his strategy was a best reply against the strategy used by the other player.

To incorporate loss aversion, the impulses are not calculated with the original payoffs but with transformed ones. In games with two strategies and a mixed Nash equilibrium each strategy has a minimal payoff and the maximum of the two minimal payoffs is called the pure strategy maximin. This pure strategy maximin is the maximal payoff a player can obtain for sure in every round and it forms a natural aspiration level. Amounts below this aspiration level are perceived as losses and amounts above this aspiration level are perceived as gains. In the case of our cyclic games the payoff for not entering a market $W$ forms this aspiration level. In line with prospect theory (Kahneman and Tversky 1979) losses are counted double in comparison to gains. ${ }^{2}$

\footnotetext{
${ }^{2}$ We consider impulse-balance equilibrium as a concept, which is free of parameters. Keeping the lossaversion fixed to 2 allows impulse-balance equilibrium to be used for predictions in different games without estimating a parameter. This is a big advantage over parametric concepts for which the parameter must be
} 

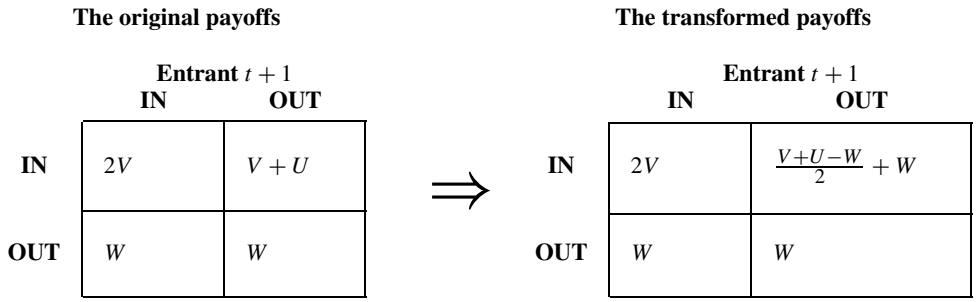

Fig. 2 Entrant $t$ 's original and transformed payoffs for an occupied market, assuming that $U+V>$ $W>2 V$ is fulfilled

Thus, gains (the part above $W$ ) are cut into half for the computation of impulses. Figure 2 illustrates this transformation for the investigated cyclic oligopoly games.

Impulse-balance equilibrium is reached at a point in which the expected impulses in both directions are equal. Let $\alpha$ be the probability of entering an occupied market at impulse-balance equilibrium. If the potential entrant enters the market he receives no impulse if the next player does not enter, since in this case entering proved to be the best choice, ex-post. If the other player also enters he receives an impulse of $W-2 V$ towards OUT. Since $\alpha$ is both players' probability for entering, he receives an impulse for OUT with probability $\alpha^{2}$.

We now consider the case that the player does not enter. In this case he does not receive any feedback. Of course he knows that the next player will enter the market since it is a dominant strategy, but this is not the relevant feedback impulse-balance equilibrium relies on. In the original concept of impulse-balance equilibrium impulses are formed with players deciding simultaneously. The sequential move structure of the cyclic game leads to the problem that the next player can condition his decision on the previous player's decision and thus an ex-post reflection keeping the matched player's decision fixed is impossible. The potential entrant is interested in what the next player would hypothetically have done if he, the potential entrant, had entered the market. Therefore he assumes that the next player finds himself in the same situation of an occupied market as he himself did and performs the same action as he did in this case.

For the proportion of cases in which the potential entrant did not enter and the following player would not have entered given that the potential entrant had entered, he receives an impulse of $\left(W+\frac{V+U-W}{2}\right)-W=\frac{V+U-W}{2}$ towards IN. This happens with probability $(1-\alpha)^{2}$. Figure 3 illustrates the possible impulses.

At equilibrium the mathematical expectation of impulses in the direction of not entering is equal to the one towards entering. This is expressed by the following impulse-balance equation:

$$
\alpha^{2}(W-2 V)=(1-\alpha)^{2} \frac{V+U-W}{2}
$$

adjusted, to the whole population or parts of it, after the behavior has been observed. However, one might want to use impulse-balance equilibrium to organize the data ex-post and therefore it might be appropriate to estimate the loss-aversion directly from the data. 


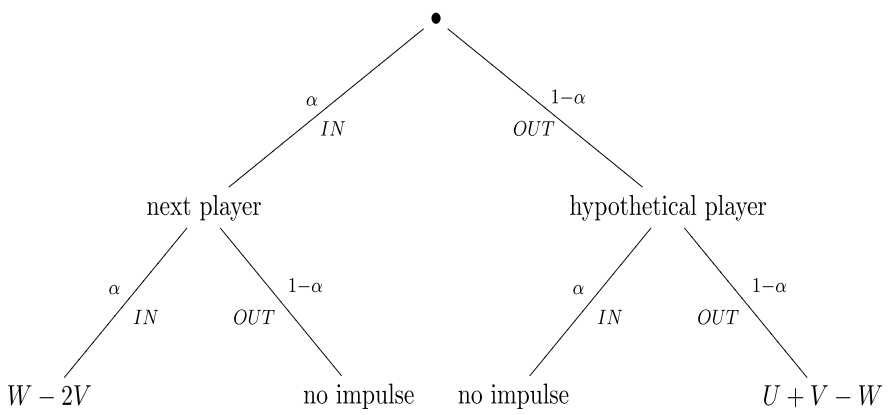

Fig. 3 Impulses in the direction of the other strategy

Thus, the probability of entering an occupied market can be stated as:

$$
\alpha=1-\frac{\sqrt{2(W-2 V)}}{\sqrt{V+U-W}+\sqrt{2(W-2 V)}}
$$

At equilibrium a player enters an occupied market with probability $\alpha=0.622$ in Game A and with $\alpha=0.449$ in Game B. In the case of a free market, impulse-balance leads to the same dominant strategy IN as the Nash equilibrium does. In addition the pure-strategy Nash equilibria are also impulse-balance equilibria, since in a pure strategy equilibrium nobody receives any impulse and therefore does not change his strategy.

The last stationary concept we will report on is the payoff-sampling equilibrium. Osborne and Rubinstein (1998) introduced this concept. The concept assumes that a player acts in accordance with previous experiences. Therefore he accesses experience in his memory made for each possible action. Thereby the number of recalled experiences is limited and the number of sampled experiences can be interpreted as the intensity used to search through the memory. ${ }^{3}$ In addition, the selection of experiences is done randomly. The order how they come to mind is haphazard and not deterministic. Afterwards the player chooses the action, which has the highest outcome given the sampled experiences. This procedure describes behavioral patterns exhibited by subjects who do not make use of information about the actions and payoffs of their opponents.

Applied to our cyclic games this means that the player draws two samples of equal sizes $n$ from previous periods, one for the strategy IN and one for the strategy OUT. He then forms the payoff sums in the two samples and compares them and plays the strategy with the highest payoff sum. If both payoff sums are equal he flips a coin and thus chooses a pure strategy with probability $\frac{1}{2}$. This rule is not part of the original concept; Osborne and Rubinstein (1998) did not discuss the case of equal payoffs.

The probability $\alpha$ for entering in the cyclic games is determined as follows. Consider a sample of $n$ cases in which the player has played IN. Let $k$ be the number of

\footnotetext{
${ }^{3}$ Osborne and Rubinstein (1998) interpret the sample size as the detailedness of the players' reasoning process or as the players' sophistication.
} 
cases in this sample in which the next player entered. Let $S_{k}$ be the payoff sum of the sample. Then we have

$$
S_{k}=(V+U)(n-k)+2 V k
$$

This payoff sum $S_{k}$ must be compared to the payoff sum $W \cdot n$ obtained for not entering $n$ times. The player does not enter if the payoff sum difference

$$
D_{k}=W n-S_{k}=(U-V) k-(V+U-W) n
$$

is positive. In the case $D_{k}=0$ the probability of not entering is $\frac{1}{2}$. The conditional probability of not entering if there are $k$ cases of next players entering in the sample for IN is as follows

$$
\eta(n, k)= \begin{cases}0 & \text { for }(V+U-W) n>(U-V) k \\ \frac{1}{2} & \text { for }(V+U-W) n=(U-V) k \\ 1 & \text { for }(V+U-W) n<(U-V) k\end{cases}
$$

With the help of this notation we now can derive an equation for the entry probability $\alpha$ :

$$
\alpha=\max \left[1-\sum_{k=0}^{n} \eta(n, k)\left(\begin{array}{l}
n \\
k
\end{array}\right) \alpha^{k}, 0\right]
$$

The sum on the right-hand side of this equation is the total probability of not entering. The probability of not entering if there are exactly $k$ cases with next players entering in the sample for IN is $\eta(n, k)$ times the binomial probability for $k$ out of $n$ players entering.

Figure 4 gives the functions of $\alpha$ for both cyclic games and shows that in both games the equation for $\alpha$ has exactly one solution in $0 \leq \alpha \leq 1$.

It is not immediately clear whether a strict pure-strategy equilibrium can be considered a payoff-sampling equilibrium. If really always only the equilibrium strategy has been played in the past, there is no sample for the other strategy. On the other hand, a learning process applying the idea of payoff-sampling may very well converge towards the pure-strategy equilibria.
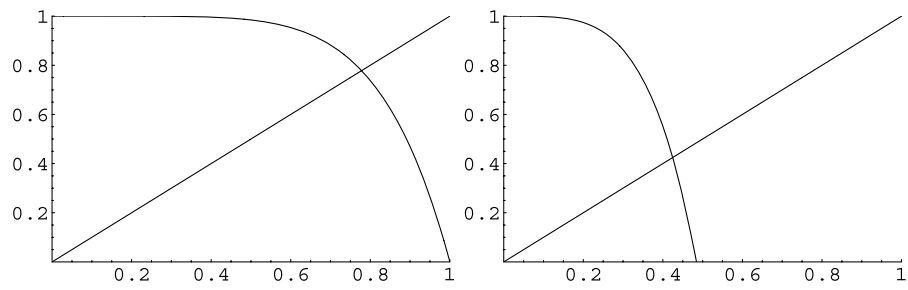

Fig. 4 Probability $\alpha$ for the payoff-sampling equilibrium in Game A (left) and Game B (right) 
Table 2 Predicted probabilities for entering an occupied market by the three different concepts, for even and uneven players

\begin{tabular}{lll}
\hline & Game A & Game B \\
\hline Nash equilibrium & 0.875 & 0.571 \\
Impulse-balance equilibrium & 0.622 & 0.449 \\
Payoff-sampling equilibrium & 0.778 & 0.424 \\
\hline
\end{tabular}

Table 2 gives the equilibria of the different concepts for both games. ${ }^{4}$ In the following we will use this probabilities as predictions for the average entry rates.

\section{Experimental design}

The experiments were carried out in 2005 (Game A) and 2008 (Game B) at the Laboratory for Experimental Economics of the University of Bonn (BonnEconLab). Seven sessions were conducted, in two sessions there were 24 participants, in four sessions there were 18 participants and in one session only twelve. In each session subjects were subdivided into independent matching groups of six. Thus we gathered a total of 22 independent observations with 11 observations per game.

Altogether 132 subjects participated in our experiment. The participants, all students, were invited via the $\mathrm{ORSEE}^{5}$ database of the laboratory. The participants came from different faculties with most of them majoring in economics (around 37\%) and law (around 21\%).

The theoretical game situation described above extends over an infinite number of time periods. However, in an experiment one cannot play for infinite time. Therefore, our experiments run over 200 rounds. The calculation of somebody's payoff, who enters in the last period, requires the decision of a potential entrant in the next period. This creates an "end-problem". We solved this problem by substituting a randomlychosen decision of an earlier entrant facing an occupied market for the decision in the next round.

Although the experiment lasted over 200 rounds each subject had to make only 100 decisions. This is due to the fact that only one half of the subjects decided in uneven rounds and the other half in even rounds.

At the beginning of the experiment the participants were briefed with written instructions, ${ }^{6}$ which were read out to them. They were informed about the duration of the experiment and that one half would decide in uneven and the other in even rounds. Furthermore they were told that before each decision each subject would be randomly matched to a market. The participants did not know that they were subdivided into independent subject groups of six. Therefore they were led to believe that there were

\footnotetext{
${ }^{4}$ The probabilities for payoff-sampling equilibrium are already given for the optimal sample size as determined in Sect. 5.1.

${ }^{5}$ See Greiner (2004).

${ }^{6} \mathrm{~A}$ translated version of the instructions can be found in the Appendix (included as a Electronic Supplementary Material).
} 
more markets to which they could be assigned. After the introduction the participants were separated into cabins with computer terminals and the experiment was started. ${ }^{7}$

On the screens ${ }^{8}$ participants first received the current status of the matched market, i.e., if the market was free or occupied. Given the status of the market they were asked whether they wanted to enter this market or not. After their decision and the decision of the next matched player they were shown their payoff for the two rounds. If they entered the market they additionally received the information whether the next matched player had entered the market or not. If they had not entered the market this information was not provided.

The payoffs in the game were given in the fictitious currency Taler and at the end of the game transferred into Euro with an exchange rate of 1 Taler equals 1 EuroCent. In addition to the cumulated payoffs subjects received a show-up fee of 5 Euro. To guarantee anonymity of the decisions participants were separately payed. Overall one session lasted about one hour and the payoffs were between 10 and 23 Euros.

\section{The experimental results}

The three concepts serve as predictions for the frequencies for entering a free market and an occupied market. In the following we do not assume that the theories can predict the behavior of a single player, but we will compare the predictive power of the three concepts for the average behavior in independent subject groups. For each independent subject group $i$ we will use the quadratic distance

$$
Q_{i}=\left(\alpha-f_{i}\right)^{2}
$$

between the theoretical probabilities $\alpha$ and the observed mean relative frequency $f_{i}$ as the measure of predictive success. The overall predictive success is measured by the mean of all theses quadratic distances over the 22 subject groups.

$$
Q=\frac{1}{22} \sum_{i=1}^{22} Q_{i}
$$

In the following we will first search for the sample size $n$ of the payoff-sampling equilibrium with the best fit to the data. Then we will compare the predictive success of the three theories. We will compare the overall predictive success and whether this success changes over time. Afterwards we will analyze tendencies of convergences towards the pure-strategy equilibria.

\subsection{Comparison of sample sizes for payoff-sampling equilibrium}

In the study by Selten and Chmura (2008) the sample size 6 yielded the best fit for the data in the twelve $2 \times 2$ games. However, it is not clear whether this sample size would lead to the predictions with the best fit to the data in our cyclical game. Therefore, we compared the predictive success of different sample sizes. We searched for one sample size, which minimizes the quadratic distance over both games.

\footnotetext{
${ }^{7}$ The experiment was programmed with z-Tree (Fischbacher 2007).

${ }^{8}$ Screenshots are shown in the Appendix.
} 


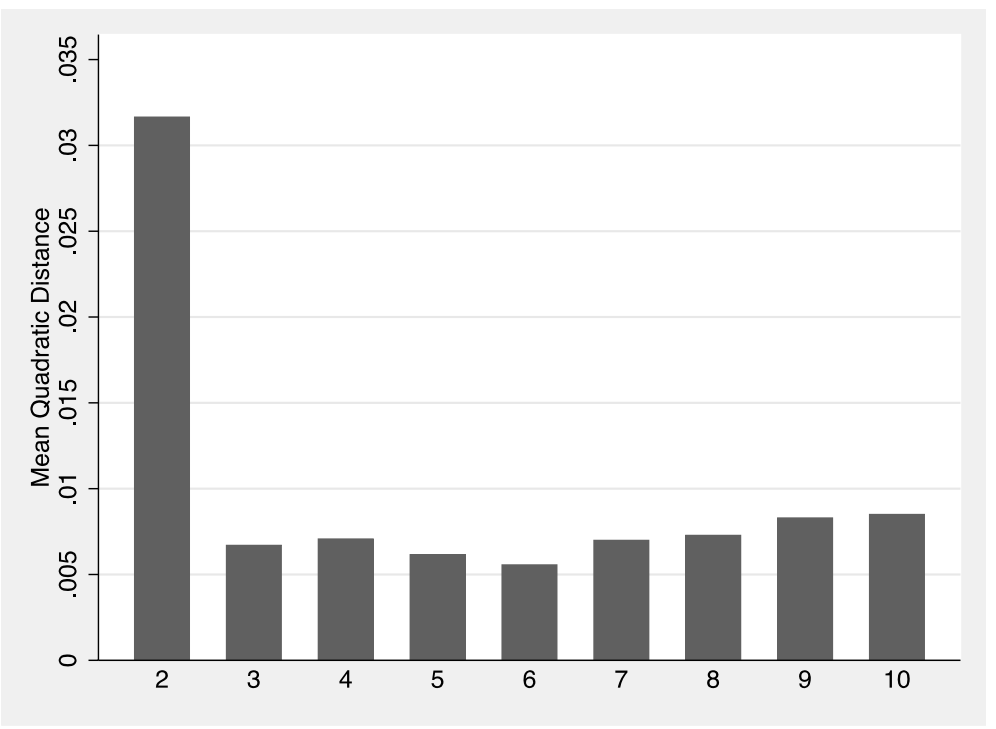

Fig. 5 Mean quadratic distance for different sample sizes

Figure 5 shows the quadratic distances for the payoff-sampling equilibrium with the sample sizes $n=2, \ldots, 10$. It can be seen that, as in Selten and Chmura (2008), the sample size 6 yields the best fit to the data. Therefore we will base our comparison of the stationary concepts on the payoff-sampling with the sample size of $6 .{ }^{9}$

\subsection{Predictive power of the three concepts}

We will start with the comparison of the relative frequencies obtained in our experiment with the predictions of the three concepts in the case of an empty market. In this case all three concepts predict a frequency of 1 for entry. In nine of the $22 \mathrm{ob}-$ servations this prediction is correct and all potential entrants join the markets when they are empty. In the other 13 observations the relative frequencies for entry are very high, too. The smallest entry rate is 0.9506 . It is not surprising that the participants realize that the strategy of not entering the market is dominated in this case by the strategy of entering the market. The numerical values for all observations are shown in Table A.3 in the Appendix.

If the market is occupied, entry is no longer the dominant strategy and different frequencies of entering are observed. In Game A the mean frequency of entering an occupied market is 0.7415 and in Game B it is 0.4224 . In the case of an occupied market the three stationary concepts predict different relative frequencies of entry. Therefore they perform differently in describing the experimental data. To measure

\footnotetext{
${ }^{9}$ Estimating the best-fitting parameter for each game separately leads to an optimal sample size of $n=5$ for Game A and to an optimal sample size of $n=9$ for Game B. Table A.1 in the Appendix gives the quadratic distances for each game and for each sample size.
} 


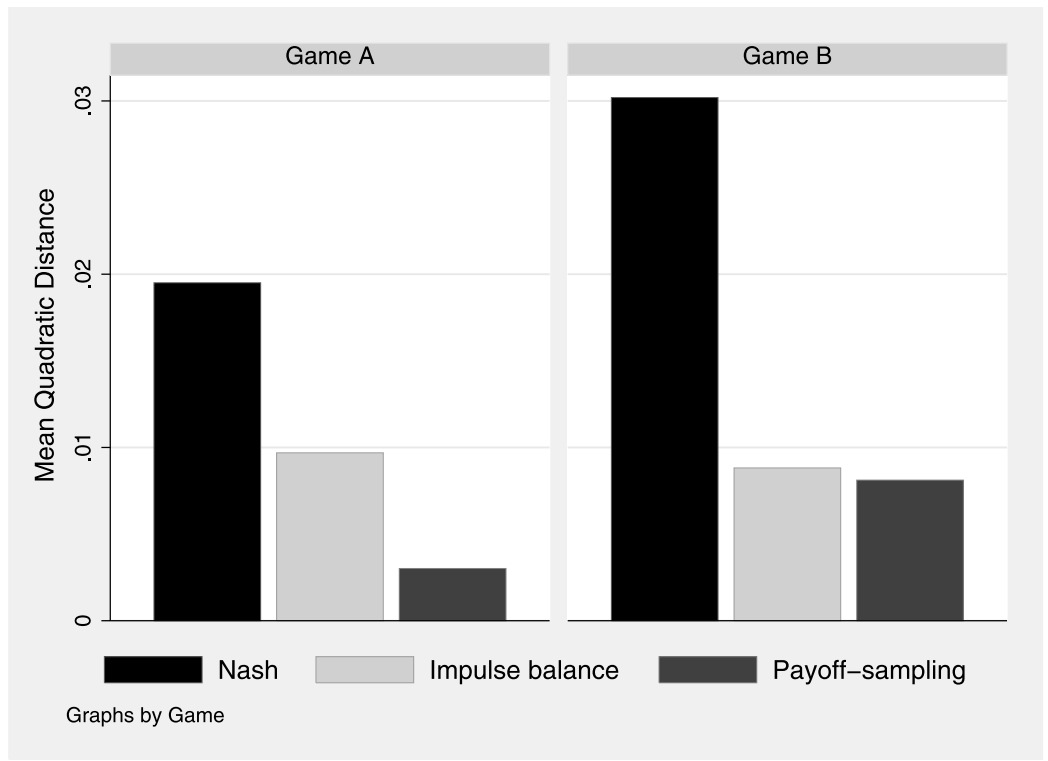

Fig. 6 Quadratic distances in occupied markets

the predictive power of each concept we use the mean quadratic distance. Figure 6 gives the mean quadratic distances to the data for each of the three stationary concepts in both games.

Obviously payoff-sampling equilibrium has the highest predictive success: In both games, payoff-sampling equilibrium has the smallest mean quadratic distance. Impulse-balance equilibrium, has in both games, the second highest quadratic distance and Nash equilibrium always has the highest quadratic distance. Table A.4 in the Appendix shows the observed frequencies and the quadratic distances of the three concepts for each observation in both games.

In 15 of the 22 observations the quadratic distance to payoff-sampling equilibrium is the smallest, in five observations the quadratic distance to impulse-balance equilibrium is the smallest and in two observations the quadratic distance to the Nash equilibrium is the smallest. In addition to this, the quadratic distance to the Nash equilibrium in three observations is smaller than the quadratic distance to the impulsebalance equilibrium, but nevertheless larger than the quadratic distances to the payoffsampling equilibrium. In 17 out of 22 observations the quadratic distance to the Nash equilibrium is the largest of all three concepts. Taking the mean quadratic distance over both games we receive a clear order of predictive success: Payoff-sampling performs the best (0.0056), impulse-balance equilibrium performs second-best (0.0092) and Nash equilibrium has the worst performance (0.0248).

Testing the quadratic distances of the three concepts with the two-sided Wilcoxon signed-rank test, we obtain the results given in Table 3 . The significances are in favor of the row concept. The first row in each cell shows the results only if the predictive power in Game A is compared, the second row only if the predictive power in Game B is compared and the third row if the predictive power over both games is compared. 
Table $3 p$-values of the two-sided Wilcoxon signed-rank test with the quadratic distances to the data in favor of the row concept. The first row gives the comparison for Game A, the second row for Game B and the last row over both games

\begin{tabular}{lll}
\hline & $\begin{array}{l}\text { Nash } \\
\text { Equilibrium }\end{array}$ & $\begin{array}{l}\text { Impulse-balance } \\
\text { equilibrium }\end{array}$ \\
\hline Payoff- & $1 \%$ & $5 \%$ \\
sampling & $2.5 \%$ & n.s. \\
equilibrium & $1 \%$ & $5 \%$ \\
Impulse- & n.s. & \\
balance & $1 \%$ & - \\
equilibrium & $1 \%$ & \\
\hline
\end{tabular}

No statistically significant difference between the predictive power of the impulsebalance equilibrium and the Nash equilibrium could be observed for Game A. But for Game B and the overall comparison, impulse-balance equilibrium fits the data significantly better than the Nash equilibrium does. Payoff-sampling equilibrium has a significantly higher predictive success than Nash equilibrium over all games and in each of the two games. The comparison between payoff-sampling equilibrium and impulse-balance equilibrium is not statistically significant in the case of Game B. In case of Game A and for the overall comparison, payoff-sampling equilibrium has a significantly higher predictive success than impulse-balance equilibrium.

But how sensitive is this order with respect to the sample size of payoff-sampling equilibrium? The comparison of payoff-sampling equilibrium and Nash equilibrium is very robust across different sample sizes. Thus, for each sample size $10 \geq n>2$, payoff-sampling equilibrium performs significantly better than Nash equilibrium in the overall comparison and in Game A. In Game B, payoff-sampling equilibrium performs significantly better than Nash equilibrium for all sample sizes except for $n=2, n=5$ and $n=10$.

In Game A, the comparison of payoff-sampling equilibrium with impulse-balance equilibrium is robust with respect to the sample size: for each sample size $10 \geq n>2$ payoff-sampling equilibrium performs significantly better than impulse balance equilibrium. In Game B, all comparisons with impulse-balance equilibrium for $10 \geq$ $n>2$ are insignificant. ${ }^{10}$ Taking both games into account, payoff-sampling equilibrium has a significant higher predictive success than impulse-balance equilibrium for $\frac{1}{3}$ of the investigated sample sizes, namely $n=3, n=6$, and $n=9$. The other investigated sample sizes do not yield significant differences. ${ }^{11}$

\subsection{Changes over time}

Up to here we have analyzed the data on an aggregated basis and could elicit an order for the predictive success for 200 periods. We now investigate whether this order

\footnotetext{
${ }^{10}$ For $n=2$ impulse-balance equilibrium performs always significantly better than payoff-sampling equilibrium and in Game B even Nash equilibrium performs significantly better than payoff-sampling with $n=2$.

${ }^{11}$ The results for all comparisons with the Wilcoxon signed-rank test are given in Table A.2 in the Appendix.
} 


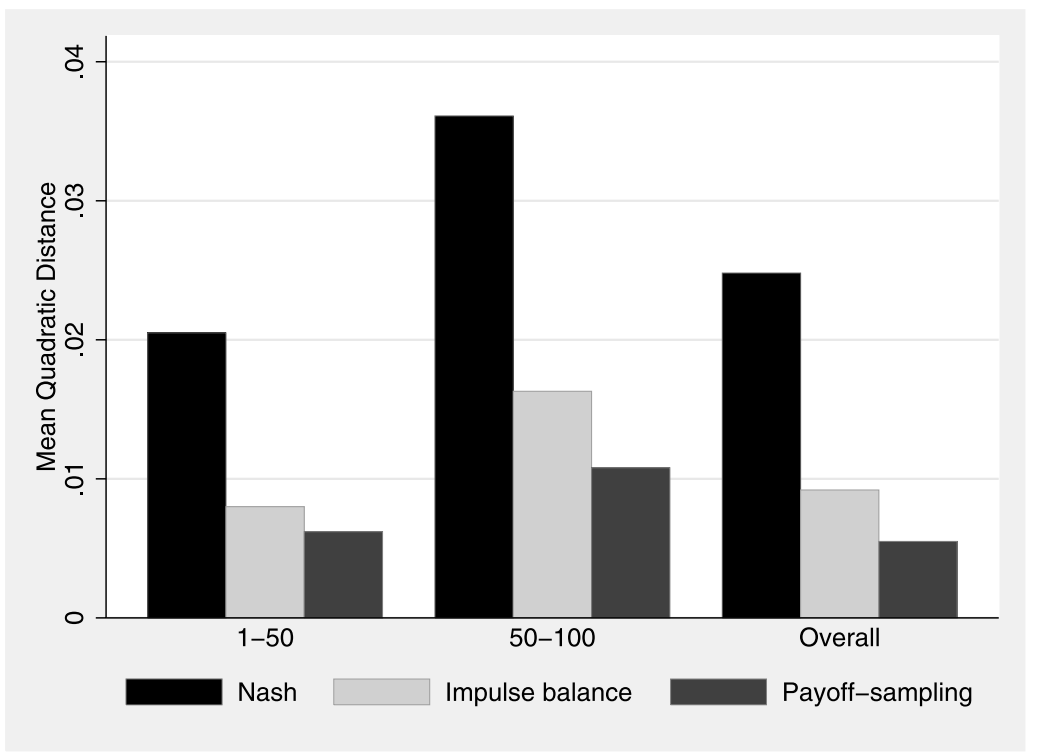

Fig. 7 Quadratic distances to the data for the first 50 decisions, the second 50 decisions, and overall

changes over time. In our experiment players had to decide whether to enter or not to enter the market 100 times. Thus, our analyses are limited to these 100 decisions. We compare the first 50 decisions with the second 50 decisions. On average, in Game A entry rates for an occupied market are rather stable over time (first half: $73 \%$; second half: $74 \%$ ), while they drop in Game B over time (first half: 46\%; second half: $37 \%$ ). Figure 7 gives the mean quadratic distance of the three theories to the observed frequency of entering the market for the first 50 decisions, for the second 50 decisions, and for all 100 decisions.

The mean quadratic distance of all three concepts increases over time. The increase of the quadratic distance over time of impulse-balance equilibrium and the payoff-sampling equilibrium is not significant, neither for one of the two games nor for all 22 observations (Wilcoxon signed-rank test). The declined predictive success over time of the Nash equilibrium is neither significant over all games and in Game A, ${ }^{12}$ but in Game B it is weakly significant ( $p=0.1$, two-sided Wilcoxon signed-rank test).

The increased quadratic distances of the three stationary concepts to the experimental data is at least partly caused by an inertia. The number of strategy changes between periods decreases over time. For the first 50 decisions in Game A $25.97 \%$ (Game B 22.67\%) of the chosen action differed from the round before. In the second 50 decisions this fraction dropped in Game A to $19.18 \%$ (Game B 13.39\%). There is only one independent observation group in Game A and two in Game B in which the number of strategy changes increases over time. Overall, there are significantly

\footnotetext{
${ }^{12}$ In fact, the quadratic distance of the Nash equilibrium decreases over time in Game A, but it is only a minor effect and not statically robust.
} 
Table $4 p$-values of the two-sided Wilcoxon signed-rank test with the quadratic distances of the data in rounds $1-50$ (top) and rounds 51-100 (bottom) in favor of the row concept

\begin{tabular}{|c|c|c|}
\hline & $\begin{array}{l}\text { Nash } \\
\text { equilibrium }\end{array}$ & $\begin{array}{l}\text { Impulse } \\
\text { balance } \\
\text { equilibrium }\end{array}$ \\
\hline Payoff-sampling & 0.005 & n.s. \\
\hline equilibrium & 0.001 & 0.05 \\
\hline Impulse-balance & 0.025 & - \\
\hline equilibrium & 0.01 & \\
\hline
\end{tabular}

less changes of actions in the second half of the game ( $p<0.01$, two-sided Wilcoxon signed-rank test).

We now turn our attention to the comparison between the three concepts over time. Table 4 shows the two-sided significances of the Wilcoxon signed-rank test in favor of the row concept. The first value in a cell is the level of significance in the first 50 decisions and the second value is the level of significance in the second 50 decisions.

Comparing the behavioral concepts with the Nash equilibrium, the significance levels are a little bit weaker in the first 50 decisions than in the second, but tendencies are the same as in the overall comparison. The prediction of the Nash equilibrium has a significantly weaker predictive success than the predictions of impulse-balance equilibrium and of the payoff-sampling equilibrium. No significant difference can be found for the comparison of the predictive success of impulse-balance equilibrium and payoff-sampling equilibrium for the first 50 rounds. Over time the difference between higher predictive success of the payoff-sampling equilibrium in comparison to the one of impulse-balance equilibrium becomes significant.

\subsection{Convergence to pure strategies?}

Altogether, 7 out of 66 subjects in Game A and 6 out of 66 subjects in Game B always entered the market. If only the last 50 decisions are analyzed, the number of participants who always entered the market increases to 18 participants in Game A and 15 in Game B.

In the above analysis we compared the mean entrance frequencies with the prediction of the three concepts in mixed strategies. However, there are also further asymmetric equilibria in pure strategies. In these equilibria one player always enters an occupied market whereas the other never does. Of course at equilibrium the first type of player never gets an opportunity to enter an occupied market but nevertheless it is his strategy to do this if he can. In our experimental setup coordination at an asymmetric pure equilibrium is possible, since one type of players decides to enter a market or not in odd periods and the other one in even periods.

As mentioned there exist a remarkable portion of players who always enter the market, but the number of players who never enter is extremely small. Over all periods there is no single player who never entered an occupied market and for the last 50 decisions only 1 player in Game A and 3 players in Game B did so. Not enough players choose such low entry rates that the predictive power of the pure-strategy 
Table 5 Quadratic distance between entry in odd and even periods

\begin{tabular}{lllll}
\hline Observation & Game A & Game B & $51-100$ \\
\cline { 2 - 4 } & $1-50$ & $51-100$ & $1-50$ & 0.6834 \\
1 & 0.0007 & 0.0067 & 0.0536 & 0.8711 \\
2 & 0.0002 & 0.1444 & 0.3155 & 0.2008 \\
3 & 0.0097 & 0.0940 & 0.0678 & 0.6765 \\
4 & 0.0025 & 0.1182 & 0.3642 & 0.4799 \\
5 & 0.0125 & 0.0072 & 0.1326 & 0.0700 \\
6 & 0.0465 & 0.1764 & 0.0518 & 0.3202 \\
7 & 0.0312 & 0.0933 & 0.0360 & 0.1729 \\
8 & 0.1044 & 0.0614 & 0.0526 & 0.0209 \\
9 & 0.0021 & 0.0982 & 0.2136 & 0.3184 \\
10 & 0.0044 & 0.0281 & 0.3141 & 0.2872 \\
11 & 0.0405 & 0.0058 & 0.0463 & 0.3728 \\
\hline Mean & 0.0231 & 0.0758 & 0.1498 &
\end{tabular}

equilibria can compete with the mixed Nash equilibria, mixed impulse-balance equilibria and the mixed payoff-sampling equilibria. But nevertheless it is quite possible that learning processes produce a tendency towards a coordination at an asymmetric pure equilibrium. In the following we shall argue that a tendency in this direction can be observed in our data and that eventually in longer experiments this process might lead to the pure-strategy equilibria.

The decrease of changes, as discussed in the previous section, might be a sign for a convergence towards the pure-strategy equilibria, in which one player type always enters an occupied market and the other one never does. Of course it cannot be predicted how the roles in the asymmetric equilibria will be distributed to the players deciding in odd and even rounds. However, we can compare the relative frequencies of entries into occupied markets for players deciding in odd and even periods in the first 50 and the second 50 decisions. Therefore we form the quadratic distance between the entrance rates in even and odd periods. Table 5 gives these quadratic distances for the first and second 50 rounds per observation and game.

In 18 of the 22 observations the quadratic distance of entries in odd and even rounds increased over time, as the mean distance over all observations did. A tendency towards coordination to a pure-strategy equilibrium would lead to such an increase. The two-sided Wilcoxon signed-rank test between the quadratic distances in the first and second 50 decisions over both games reveals a highly significant difference with $p=0.002$.

One realizes that the quadratic distances between even and odd rounds are higher in Game B than in Game A. Recall that in Game B, not entering an occupied market is more attractive than in Game A and therefore results in lower entry rates. Lower entry rates in case of an occupied market enhance the coordination and the crowding out of potential entrants, respectively. Nevertheless, in both games the tendencies are the same and statistically significant (Game A: $p=0.04$; Game B: $p=0.01$, both two-sided Wilcoxon signed-rank test). 


\section{Summary and discussion}

In this paper three stationary concepts, namely mixed Nash equilibrium, impulsebalance equilibrium, and payoff-sampling equilibrium, have been compared in an experimental setting. The three stationary concepts had to describe the cumulated behavior in two market entry games, which were based on the structure of a cyclic game introduced by Selten and Wooders (2001).

Altogether 22 independent subject groups, eleven per game, participated in the experiment. Each independent subject group consisted of 6 participants, three deciding in odd rounds and three deciding in even rounds. Each subject group played over 200 rounds with random matching. In the case of an empty market all three theories predicted entry and nearly all participants acted accordingly.

In the case of an occupied market the three stationary concepts predict different probabilities for entry. We used the mean squared distance between predicted and observed frequencies as the measurement of the predictive success of a theory. The comparison of the mean squared distances reveals the following order, from best to worst: payoff-sampling equilibrium, impulse-balance equilibrium and mixed Nash equilibrium. Pairwise testing shows that this order is statistically robust.

In addition to the mixed equilibrium the game also has two pure equilibria, in which players deciding in odd periods always enter and those deciding in even periods never enter, or vice versa. We observe a weak tendency towards these equilibria, as the quadratic distance between the relative frequencies of entering in odd and even periods is significantly higher in the second half of the experiment than in the first one.

A further sign for this tendency is the number of strategy changes, which decreases significantly over time. This inertia leads to a decreasing predictive success of the three stationary concepts over time. For the second 50 decisions payoff-sampling equilibrium performs significantly better than impulse-balance equilibrium. This is connected to the inertia and a pronounced drop of the entry rates in Game B, in which payoff-sampling equilibrium predicts the lowest entry rates.

Nevertheless, the observed frequencies are far from those predicted by the pure equilibria and all mixed stationary concepts describe the data more accurately than the pure-strategy equilibria. Our findings give support to the results of Selten and Chmura (2008) where impulse-balance equilibrium and payoff-sampling equilibrium outperformed the mixed Nash equilibrium. In contrast to Selten and Chmura (2008) payoff-sampling performs significantly better in our cyclic duopoly game than impulse-balance equilibrium.

A plausible reason for this result might be the sequential move structure, which makes the calculations of impulses for the case of not entering a market not as intuitive as the general calculation of impulses in the $2 \times 2$ games. A player never experiences an impulse, if he does not enter. In this case only hypothetical impulses can be constructed on the basis of the idea that the other player follows the same decision process as one does oneself. Therefore the partial lack of feedback makes impulse-balance theory less applicable to cyclic games. While the calculation of impulses is no longer as intuitive as for the $2 \times 2$ games, the drawing of payoff-samples is not affected by the sequential move structure. Therefore payoff-sampling equilibrium might be the favorable approach to games with sequential moves. This line of 
reasoning is supported by our observations from Game A, in which payoff-sampling equilibrium performed significantly better than impulse-balance equilibrium for all sample-sizes $n>2$.

A second reason for the better performance of payoff-sampling might be the parameter of the sample sizes, which represents the detailedness of, or the effort for, the underlying process of memorization. The free parameter might work in favor of the payoff-sampling equilibrium. This thesis is supported by our overall comparison and by the fact that over both games, payoff-sampling equilibrium performed significantly better with only one third of the investigated sample sizes than impulse balance equilibrium.

Besides this, the question arises why the models with bounded rationality outperform the concept of Nash equilibrium. While it is known that learning dynamics based on Bayesian updating might lead to a Nash equilibrium (e.g., Kalai and Lehrer 1993 ) it is by no means clear that actual human learning mechanisms must converge to Nash equilibrium. Our results suggest that at least for the short term of 200 rounds human learning processes approach different points. ${ }^{13}$

We believe that our results might enrich various economic fields working with stationary concepts to describe, for example, the behavior of market participants, the behavior in industrial organizations, the behavior in binary decisions and the behavior in bargaining situations. After all, behavioral stationary concepts might give models a higher accuracy in describing the distribution of human behavior.

Acknowledgements Financial support by the Deutsche Forschungsgemeinschaft is gratefully acknowledged. In addition we would like to thank Jordi Brandts and two anonymous referees for providing very helpfull comments.

Open Access This article is distributed under the terms of the Creative Commons Attribution Noncommercial License which permits any noncommercial use, distribution, and reproduction in any medium, provided the original author(s) and source are credited.

\section{References}

Avrahami, J., Kareev, Y., \& Güth, W. (2005). Games of competition in a stochastic environment. Theory and Decision, 59(4), 255-294.

Brown, J., \& Rosenthal, R. (1990). Testing the minimax hypothesis: A re-examination of O’Neill's game experiment. Econometrica, 58, 1065-1081.

Diamond, P. A. (1965). National debt in a neoclassical growth model. The American Economic Review, 55(5), 1126-1150.

Erev, I., \& Roth, E. A. (1998). Predicting how people play games: reinforcement learning in experimental games with unique mixed strategy equilibria. American Economic Review, 88(4), 848-881.

Fischbacher, U. (2007). z-tree: Zurich toolbox for readymade economic experiments. Experimental Economics, 10(2), 171-178.

\footnotetext{
${ }^{13}$ Of course, stationary concepts are not the optimal approach to individual period-by-period behavior, but the underlying ideas of impulse-balance equilibrium and payoff-sampling equilibrium contain precise descriptions of behavior. Therefore they are predestined to be used as the basis of learning models. Further studies should investigate whether those learning models are able to capture subjects' tendency towards the equilibria in pure strategies.
} 
Greiner, B. (2004). An online recruitment system for economic experiments. In K. Kremer \& V. Macho (Eds.), GWDG Bericht 63. Forschung und wissenschaftliches Rechnen 2003 (pp. 79-93). Goettingen: Ges. fuer Wiss. Datenverarbeitung.

Kahneman, D., \& Tversky, A. (1979). Prospect theory: an analysis of decision under risk. Econometrica, 47(2), 263-291.

Kalai, E., \& Lehrer, E. (1993). Rational learning leads to Nash equilibrium. Econometrica, 61(5), 10191045.

Osborne, M. J., \& Rubinstein, A. (1998). Games with procedurally rational players. American Economic Review, 88(4), 834-847.

Selten, R., \& Buchta, J. (1999). Experimental sealed bid first price auctions with directly observed bid functions. In D. Budescu, I. Erev, \& R. Zwick (Eds.), Games and human behavior: essays in the honor of Amnon Rapoport. Hillsade: Erlbaum.

Selten, R., \& Chmura, T. (2008). Stationary concepts for experimental $2 \times 2$ games. American Economic Review, 98(3), 938-966.

Selten, R., \& Wooders, M. (2001). Cyclic games: an introduction and some examples. Games and Economic Behavior, 39, 138-152.

Selten, R., Abbink, K., \& Cox, R. (2005). Learning direction theory and the winner's curse. Experimental Economics, 8(1), 5-20. 